\title{
Comparison of the microleakage between two different flowable composite resin restorations with 8th generation bond system in Class II cavity: An in-vitro stereomicroscopic study.
}

\begin{abstract}
Introduction: Marginal adaptability of composite resins is one of the prime factors for the success of class II cavity restoration.

Materials and Methodology: Forty non-carious extracted mandibular molar with fully formed apices were collected and Class II box type cavities were prepared on both mesial and distal surfaces of every tooth using a new straight fissure diamond bur and high-speed airrotor handpiece. According to the type of restorative materials used, teeth were divided into Group I ( $n=20)$ : Restored with SDR (Smart Dentin Replacement) and Group II ( $n=20)$ : Restored with Tetric Eva Flow Bulk Fill. After 24 hours of storage in distilled water at $37^{\circ} \mathrm{C}$, the restored specimens were subjected to artificial aging by thermocycling. The teeth were then immersed in a $2 \%$ methylene blue dye for 24 hours. All samples were cut longitudinally through the center of the restorations with the help of an isomet diamond saw. The sections were then observed under a stereomicroscope at $25 X$ for scoring the depth of dye penetration at cervical and axial walls toward the pulp.

Results: Mean score of Group la is $0.4 \pm 0.83$ and lb was $0.55 \pm 0.88$ respectively while the mean score of Group lla was $0.75 \pm 1.118$ and in $l \mathrm{lb}$ was $0.75 \pm 1.019$ respectively. Microleakage was found to be highest in Group Ila and minimum in Group la.

Conclusion: The use of a flowable composite SDR above and below the CEJ in Class II composite resin restorations showed good results.
\end{abstract}

Keywords: Microlekage, Class II Cavity, Flowable Composites, Thermocycling.

\section{Introduction:}

An esthetically pleasing smile has always been the objective of mankind. Composite resins are being used for more than four decades as an anterior esthetic restorative material.[1] The advent of resin-based composites served as a viable toothcolored alternative to amalgam restorations.[2] In recent years, the demand for posterior resin composite restorations has dramatically increased because of their ability to match tooth color, absence of mercury, thermally nonconductive, biocompatibility, and ability to bond to the tooth structure. Although the mechanical properties and abrasion resistance of resin-based composites have improved considerably over the years, the placement of posterior resin-based restoration remains very technique sensitive. Polymerization shrinkage of the composite during curing induces stresses at the tooth restoration interface resulting in gap formation and marginal leakage.[3] Besides, the inherent differences in thermal expansion coefficients between the composite resin and the tooth structure can also contribute to marginal leakage.

\begin{tabular}{|l|c|}
\hline \multicolumn{3}{|c|}{ Access this article online } \\
\hline \multirow{2}{*}{ Website: } \\
www.ujds.in
\end{tabular}

Leakage at the tooth - restoration interface leads to staining at the margin of the restoration, recurrent caries, hypersensitivity of the restored teeth and eventually leading to pulpal pathology.[4]

With the advent of the packable composites improved layering technique with good micromechanical bonding to the enamel, has been consistently achieved. Bonding to dentin is still

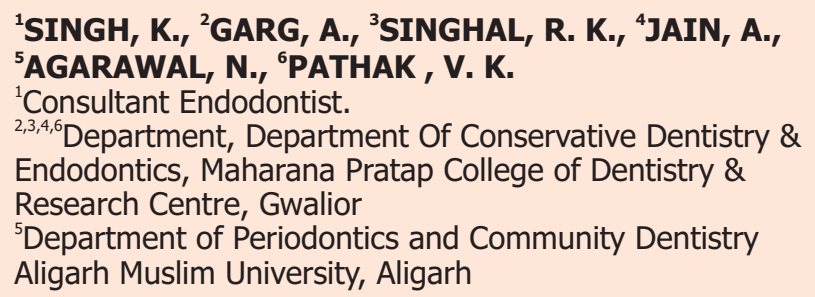

Address for Corresponding : Dr. Vivek Kumar Pathak MDS in Endodontics

Senior Lecturer, Department Of Conservative Dentistry \& Endodontics, Maharana Pratap College of Dentistry \& Research Centre, Gwalior.

Email: vivekpathak786@gmail.com

Received : 3 Nov. 2020, Published : 31 Dec. 2020 
questionable due to its higher organic content, tubular structure, fluid pressure, and lower surface energy which makes bonding a difficult procedure.[5] A systematic review was conducted by Boruziniat A et al. in 2016,[6] analyzed the efficacy of flowable composite as lining material on microleakage of composite resin restorations. They found that the use of flowable composites was advocated by various authors. Flowable composite has poor strength and wears resistance compared with packable composites. Anyway, their low viscosity expanded elasticity, and wet ability to empower them to adjust to whole dividers superior to the packable composites. The recently created bulk-fill resins offer composites including low-thickness (flowable) and high-consistency (sculptable) material types. The SDR (Smart Dentin Replacement), Posterior Bulk fill flowable base is a single component, fluoride-containing, and light relieved radiopaque resin composite therapeutic material. It has the handling characteristic of a flowable composite however can be set in $4 \mathrm{~mm}$ additions with insignificant polymerization stress. The SDR has a self-leveling highlight that allows intimate adaptation to the prepared cavity walls.

Tetric Evo Flow Bulk Fill (Ivoclar Vivadent) is a nanohybrid composite with a monomer matrix containing dimethacrylates (20-21\% weight). The fillers contain barium glass, ytterbium trifluoride, blended oxide, and prepolymer (78\% $-81 \%$ by weight). Extra substances are added substances, catalysts, stabilizers, and pigments $(<1.0 \%$ weight). The total substance of inorganic fillers is 76$77 \%$ weight or $53-54 \%$ volume. Tetric Evo Flow Bulk Fill contains in its composition an inhibitor of affectability to light and along these lines gives delayed time for modeling of filling, an inhibitor of shrinkage stress keeping in mind the end goal to accomplish ideal marginal seal, and Ivocerin, polymerization photoinitiator permitting curing of $4 \mathrm{~mm}$ layers of material.[7]

Futurabond DC (VOCO, Cuxhaven, Germany) is a self-etch bonding system that comprises two fluids. A drop of fluid A and a drop of fluid B were mixed, and afterward, the mixture was rubbed on the enamel surface for 20 seconds, air-dried, and light-relieved for 10 seconds.[8]

With this background, the present in vitro study was planned to evaluate microleakage in class II cavity restored with SDR and Tetric Evo Flow Bulk Fill using 8th generation universal adhesive system and, to compare microleakage above and below cementoenamel junction in these class II cavities.
Material and Methodology:

Forty non-carious extracted mandibular molar with fully formed apices were collected from the Department of Oral and Maxillofacial Surgery, and several other dental offices (General Dentists, Oral Surgeons, and Periodontist). The teeth were cleaned of calculus, soft tissue, and debris with hand instrumentation and stored in isotonic saline solution at $4^{\circ} \mathrm{C}$ for not more than one month after extraction. The incursion criteria of the study consisted of [1]. Teeth with completely formed root apices[2]. Multi rooted mandibular teeth[3]. Teeth without resorptive defects.[4]. Teeth without root fillings[5]. Roots without cracks. The exclusion criteria consisted of [1]. Teeth with external resorption[2]. Malformed teeth[3]. Teeth with developmental anomalies.[4]. Vertical/horizontal root fracture[5]. Endodontically treated teeth[6]. Teeth with deep carious lesions[7]. Teeth of individuals who had participated in any other clinical study.

Specimen preparation- Each specimen provided two surfaces for preparation mesial and distal. To simulate the clinical situation during restoration placement, a "restoration template" of size 3.5 " x 4.5 " was fabricated. Two mandibular molars were embedded in dental stone type III (Gyprock, Rajkot, India) to the level of the cement-enamel junction, almost $11 \mathrm{~mm}$ apart. The sample specimen was embedded between these two teeth in addition to silicone impression material (Dentsply, Ballaigues, Switzerland). Class II box type cavities were prepared on both mesial and distal surfaces of every tooth using a new straight fissure diamond bur No.012 (Mani, Tochigi, Japan) by high-speed air rotor handpiece (Being, Foshan, China). The dimensions of the preparation were $4 \mathrm{~mm}$ (buccolingual width) with a pulpal depth of $2 \mathrm{~mm}$. Cavities were then restored with a bulk-fill technique using the PalodentV3-contoured sectional matrix (Dentsply, Ballaigues, Switzerland).

According to the type of material used the teeth were divided into two groups:

Group I ( $\mathrm{n}=20)$ : Restored with SDR (Smart Dentin Replacement) after applying Futurabond DC. Group I was further divided into Ia and $\mathrm{Ib}$ depending upon the application of SDR above and below the CEJ respectively.

Group II ( $\mathrm{n}=20)$ : Restored with Tetric Eva Flow Bulk Fill after applying the Futurabond DC PalodentV3 matrix system and then bonding the tooth by Furturabond DC and gentle airdried for 5 seconds and cured for 10 seconds. After that 
restoring the tooth by bulk-fill technique, followed by light curing for 20 seconds. Group II was further divided into IIa and IIb depending upon the application of Tetric Eva Flow Bulk Fill above and below the CEJ respectively.

Samples were stored in distilled water for 24 hours at $37^{\circ} \mathrm{C}$. Then the restored specimens were subjected to artificial aging by thermocycling by immersing them alternatively in a water bath at $5 \pm 2^{\circ} \mathrm{C}$ and $55 \pm 2^{\circ} \mathrm{C}$ for 1500 cycles with a dwell time of $30 \mathrm{sec}$ and transfer time of 15 seconds. To prevent dye penetration into dentinal tubules and lateral canals, the apices were sealed with sticky wax, and teeth were coated with two layers of fingernail polish, except for an area approximately $1 \mathrm{~mm}$ around the margins of the restoration. The teeth were immersed in $2 \%$ methylene blue dye for 24 hours. The sticky wax was removed after the dye exposure. The treated teeth were then sectioned mesiodistally using the Diamond disk and Mandrel (DFS Diamon, Reidenburg, Germany), and dye penetration at the enamel, dentin, and cementum margins was scored under a stereomicroscope (Biocraft Model no. Bsm12) as 0- No dye penetration (Fig. no. 1).1- Dye penetration extending to $1 / 3 \mathrm{rd}$ of the cervical wall (Fig. no. 2). 2-Dye penetration extending to $2 / 3 \mathrm{rd}$ of the cervical wall (Fig. no. 3). 3- Dye penetration into the whole of the cervical wall (Fig. no. 4). 4- Dye penetration into the cervical wall and axial walls toward the pulp (Fig. no. 5). The data so obtained was compiled systematically by preparing the master table, tabulated, and subjected for statistical analysis by IBM SPSS version 21.0.

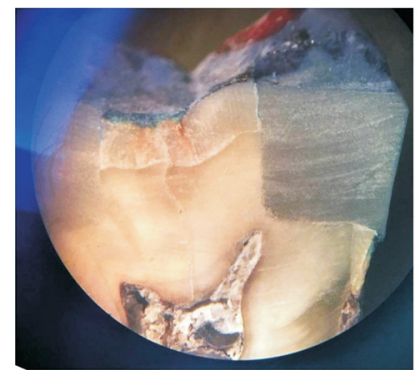

Figure No.1 Microleakage at the tooth restoration interface showing no microleakage at the interface

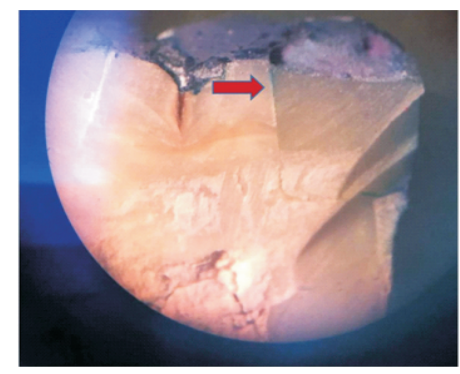

Figure No.2 : Microleakage at tooth restoration interface showing dye penctration up to $1 / 3$ rd of the cervical wall

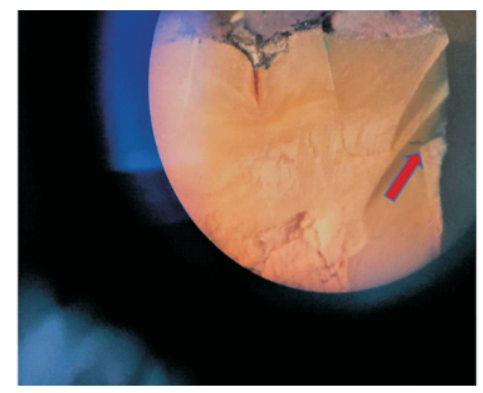

Figure No.3 : Microleakage at tooth-restoration interface dye penetration upto $2 / 3$ rd of cervical wall.

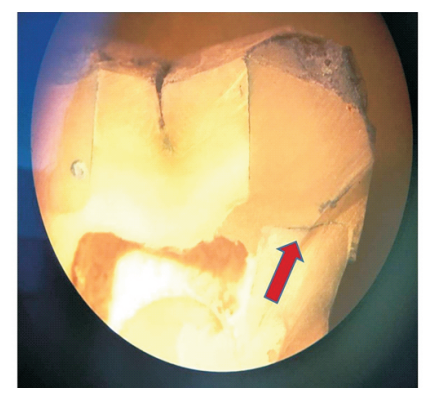

Figure No. 4 : Microleakage at tooth restoration interface dye penetration into the whole of the cervical wall.

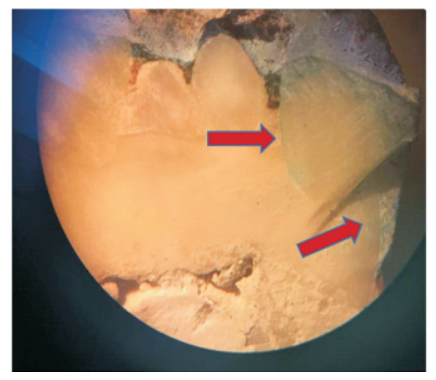

Figure No. 5 Microleakage at tooth restoration interface dye penetration into the cervical wall and axial walls toward the pulp.

\section{Results:}

Table 1 and Graph 1 showed the mean scores with standard deviation obtained in two study groups. The mean score of microleakage in Group Ia is $0.4 \pm 0.83$ and $\mathrm{Ib}$ was $0.55 \pm 0.88$ respectively while the mean score of Group IIa was $0.75 \pm 1.118$ and in IIb was $0.75 \pm 1.019$ respectively. Table 2 depicted the Mann-Whitney test (U) for the intra-group comparison. The value of $\mathrm{U}$ in Ia-Ib is 180 with a $\mathrm{z}$ value of 0.52748 and p-value $0.29806(>0.05)$ which was found to be insignificant while IIa-IIb was also found to be insignificant with $\mathrm{p}=0.48006(>0.05)$ with $\mathrm{U}=197.5 \& \mathrm{Z}=-0.0541$. Table 3 depicted the inter-group comparison which showed 
insignificant changes among all the groups. The inter-group comparison of Ia-IIa, the $\mathrm{U}=160.5, \mathrm{Z}=-1.05495$ with $\mathrm{p}=$ 0.14686 . The Group Ia-IIb showed an insignificant result with $\mathrm{U}=159, \mathrm{Z}=-1.09553$, and $\mathrm{p}=0.27134$. The Group Ib-IIa also showed an insignificant result with $\mathrm{U}=181, \mathrm{Z}=-0.50043$ with $\mathrm{p}=0.61708$. The Ib-IIb group also showed the insignificant result with $U=178.5, Z=-0.56805$ with $\mathrm{p}=0.56868(>0.05)$. Results revealed that the comparison of the mean scores in all groups was found to be highest in Group IIa $>$ Group IIb $>$ Group Ib $>$ Group Ia. Group Ia showed the minimum microleakage. The Mann-Whitney $U$ test is a nonparametric test that allows two groups or conditions or treatments to be compared without assuming that values are normally distributed.

Table no. 1: Mean values of microleakage in all study groups.

\begin{tabular}{|c|c|}
\hline Group & Mean \pm SD \\
\hline Ia & $0.4 \pm 0.83$ \\
\hline Ib & $0.55 \pm 0.88$ \\
\hline IIa & $0.75 \pm 1.118$ \\
\hline IIb & $0.75 \pm 1.019$ \\
\hline
\end{tabular}

Table no. 2: Mann-Whitney test (U) for the intra-group comparison

\begin{tabular}{|c|c|c|c|}
\hline Group & $\mathbf{U}$ & $\mathbf{Z}$ & p-value* \\
\hline Ia-Ib & 180 & -0.527 & 0.298 \\
\hline IIa-IIb & 197.5 & -0.054 & 0.480 \\
\hline
\end{tabular}

${ }^{*}$ p-value $>0.05$ is insignificant

Table no. 3: Mann-Whitney test (U) for the inter-group comparison

\begin{tabular}{|c|c|c|c|}
\hline Group & $\mathbf{U}$ & $\mathbf{Z}$ & p-value* \\
\hline Ia-IIa & 160.5 & -1.054 & 0.147 \\
\hline Ia-IIb & 159 & -1.095 & 0.271 \\
\hline Ib-IIa & 181 & -0.500 & 0.617 \\
\hline Ib-IIb & 178.5 & -0.568 & 0.568 \\
\hline
\end{tabular}

*p-value $>0.05$ is insignificant

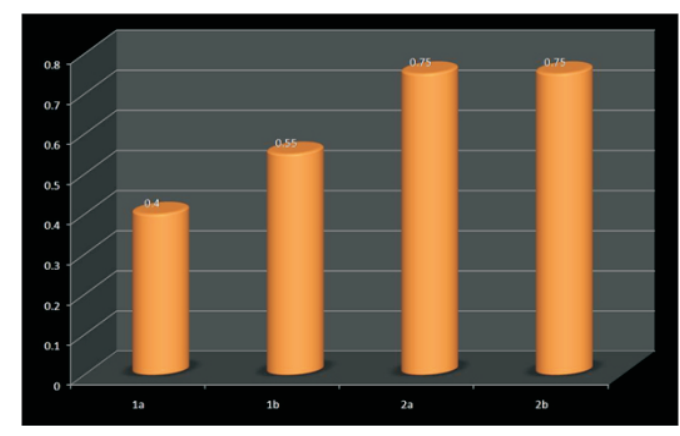

Graph1 : Comparison of mean scores

\section{Discussion:}

Composite resins introduced in the 1960s for the restoration of anterior teeth, brought about drastic improvements in the field of esthetic and adhesive dentistry. The longevity of a composite restoration is directly related to the quality of marginal adaptation. Polymerization shrinkage during composite curing induces stresses at the tooth- restoration interface resulting in gap formation leading to marginal leakage. In addition to this, the inherent differences in the thermal expansion coefficient between composite resin and the tooth structure also contribute to marginal leakage.[9]

A variety of new composite resins were introduced to the dental profession, among these, the packable composites have superior mechanical properties, high depth of cure, and low polymerization shrinkage due to which they can be used as a posterior restorative material. But even with the advent of packable composites, bonding to dentin remains questionable, due to high organic content, tubular structure, fluid pressure, and lower surface energy of dentin.[10] The poor adhesion between dentin and composite resin may predispose to gap formation leading to marginal leakage.

Studies have shown that when packable composites are placed below CEJ, there is higher marginal leakage from dentin gingival margins when compared to the cavities placed above CEJ.[11] This is because the bond strength of composite to enamel is usually higher than bond strength to dentin, as dentin is a less favorable bonding substrate. To overcome this problem studies have shown the use of flowable composite or glass ionomer cement liners in a proximal box of Class II preparation. The flowable composites are less viscous materials due to which it flows easily and adapts well to the tooth surface resulting in less leakage and post-operative sensitivity. They also serve as a flexible intermediate layer that releases stress during polymerization shrinkage of composite resin. Several studies have proved that flowable liner reduced the marginal leakage in dentinal margins (placed $1 \mathrm{~mm}$ below CEJ) in all composite restorations.[12-13] Flowable bulk-fill composites are generally designed for replacing dentin in class I and class II restorations. Their translucency should be therefore as dentinlike as possible. SDR (Smart Dentin Replacement) is a onecomponent, fluoride-containing, and visible light-cured, radiopaque resin composite restorative material. It is designed to be used as a base in class I and class II restorations. SDR material has handling characteristics typical of the flowable composite but can be placed in $4 \mathrm{~mm}$ 
increments with minimal polymerization stress. SDR material has a self-leveling feature that allows intimate adaptation to the prepared cavity walls. Available in one universal shade.[7] Tetric Evo Flow Bulk Fill is a flowable, light-curing radiopaque composite for restorations in posterior teeth as its opacity increases during polymerization. Tetric Evo Flow Bulk Fill Bulk fill is also suitable for discolored tooth structure. It is applied as an initial layer in increments of up to $4 \mathrm{~mm}$ in class I and class II restorations. Tetric Evo Flow Bulk Fill Bulk Fill cures with light in the wavelength of 400-500 nm. Tetric Evo Flow Bulk Fill Bulk Fill is available in three shades -Universal shades: IVA, IVB, and IVW. The monomer matrix is composed of dimethacrylates. The fillers contain barium glass, ytterbium trifluoride, and copolymers.[13] When an intragroup comparison is done between when flowable SDR was placed above the CEJ (Group I a) and below the CEJ (Group I b), and Tetric Evo Flow Bulk Fill was placed above the CEJ (Group II a) and below the CEJ (Group II b), there was least marginal leakage in group Ia and II $a$ than in the group I $b$ and II $b$ respectively. The probable reason for this is that the bond strength to the enamel is usually higher than the bond strength to the dentin. Dentin is a less favorable bonding substrate because of its heterogeneous structure. However, the enamel is a highly mineralized tissue with a composition of more than $90 \%$ (by volume) hydroxyapatite; dentin contains a considerable proportion of water and organic material, primarily Type I collagen. Dentin also comprises a dense network of tubules that connect the pulp with the dentinenamel junction. The tubules may branch, mainly near the amelodentinal and cement-dentinal junctions. Usually, the branching of tubules is smaller and more numerous in root dentin than in crown dentin. Acid etching of the heterogeneous dentin results in different surface chemistries and morphologies. The orientation of dentine tubules can also affect the formation of the hybrid layer.[14-17] In areas with perpendicular tubule orientation, the hybrid layer was significantly denser than areas with parallel tubule orientation. Therefore, the dentin surface on the gingival floor of class II preparations may be a surface on which a good amount of hybrid layer formation is difficult. This could well contribute to the results of the present study.[3] Group II did not perform well when compared to flowable composite in Group Ia, because Tetric Evo Flow Bulk Fill resin has volumetric shrinkage when compared to SDR composite restoration.[7] Group II showed the highest marginal leakage values when compared to other groups. Marginal leakage values are less when the flowable composite was placed in the enamel margin, than when placed in the dentinal margin of the cavity. This may be due to the effect of some primers that could have affected the adhesion between the bonding agent and enamel.[18-20]

Though there was no statistical significance in any of the groups used in this study. The best results can be obtained when flowable composite SDR is placed above the cementoenamel junction. Since this is an in vitro study further research and advancements are mandatory to determine the marginal leakage of these materials tested in a clinical situation.

\section{Conclusion:}

The study concluded that restorations with SDR (Smart Dentin Replacement) showed lesser microleakage than Tetric Eva Flow Bulk Fill after applying a flowable composite. It was also observed that the use of a flowable composite above and below the CEJ in Class II composite resin restorations showed good results. The best results can be obtained when the flowable composite is placed above the cementoenamel junction.

\section{References:}

1. Attar N, Turgut MD, Gungor HC. The effect of flowable resin composites as gingival increments on the microleakage of posterior resin composites. Oper Dent 2004;29(2):162-167.

2. Mishra P, Jaiswal S, Nikhil V, Gupta S, Jha P, Raj S. Evaluation of marginal sealing ability of self-adhesive flowable composite resin in Class II composite restoration: An in vitro study. J Conserv Dent. 2018;21(4):363-368.

3. Bogra P, Gupta S, Kumar S et al. Comparative evaluation of microleakage in class II cavities restored with Ceram $\mathrm{X}$ and Filtek P-90: An in vitro study. Contemp Clin Dent 2012;3:9-14.

4. Alani AH and Toh CG. Detection of microleakage around Dental Restorations: A Review. Oper Dent 1997;22:173185.

5. Von Fraunhofer JA and Hammer DW. Microleakage of composite resin restorations. J Prosthet.Dent 1984;51(2):209-213.

6. Boruziniat A, Gharaee S, Sarraf Shirazi A, Majidinia S, Vatanpour M. Evaluation of the efficacy of flowable composite as lining material on microleakage of composite resin restorations: A systematic review and meta-analysis. Quintessence Int. 2016;47(2):93-101. 
7. Orbowski M, tarczydbo B. Evaluation of marginal integrity of Four Bulk-Fill Dental Composite Materials: In Vitro Study. Sci World J 2015; 4:1-8.

8. Hammad SM, El-Wassefy N. Effect of nanotechnology in self-etch bonding systems on the shear bond strength of stainless steel orthodontic brackets. Dental Press J Orthod. 2017;22(1):47-56.

9. Gale MS, Darvell BW.Thermal cycling procedures for laboratory testing of dental restorations. J Dent 1999;27:89-99.

10. Yazici AR, Baseren Mand Dayangac B. The effect of flowable resin composite on Microleakage in class $\mathrm{V}$ cavities. Oper Dent 2003;28:42-46.

11. Leevailoj C, Cochran MA, Matis BA et al. Microleakage of Posterior Packable Resin Composites with and Without Flowable Liners. Oper Dent 2001;26:302-307.

12. Olmez A, OztasNand Bodur H. The effect of flowable resin composites on microleakage and internal voids in class II composite restorations. Operative Dentistry 2004;29(6):713-719.

13. Niek JM, Opdam, Bodur Het al. Necessity of bevels for box only Class II composite restorations. J. Prosthet Dent 1998; 80:274-279.

14. Meshram P, Meshram V, Palve D, Patil S, Gade V, Raut A. Comparative evaluation of microleakage around Class V cavities restored with alkasite restorative material with and without bonding agent and flowable composite resin: An in vitro study. Indian J Dent Res 2019;30:403-7

15. Punathil S, Almalki SA, AlJameel AH, Gowdar IM, Mc VA, Chinnari K. Assessment of Microleakage Using Dye Penetration Method in Primary Teeth Restored with Tooth-colored Materials: An In Vitro Study. J Contemp Dent Pract. 2019;20(7):778-782.

16. Lokhande NA, Padmai AS, Rathore VP, Shingane S, Jayashankar DN, Sharma U, et al. Effectiveness of flowable resin composite in reducing microleakage - an in vitro study. J Int Oral Health 2014;6:111-4.

17. Gonulol N, Ertas E' YI lmaz A, Soner C. Effect of thermal aging on microleakage of current flowable composite resins. J Dent Sci 2015;10:376-82 .

18. Sudha K, Mohan TM, Aparna K, Yadav BS, Rani ES, Sowjanya DL. Comparative evaluation of microleakage of conventional crowns and endocrowns using confocal laser scanning electron microscope ? An in vitro study. $\mathrm{J}$ NTR Univ Health Sci 2020;9:6- 11.

19. Somani R, Jaidka S. Comparative evaluation of microleakage of newer generation dentin bonding agents: An in vitro study. Indian J Dent Res 2016;27:86-90.

20. Mazumdar P, Das A, Das UK. Comparative evaluation of microleakage of three different direct restorative materials (silver amalgam, glass ionomer cement, cention $\mathrm{N}$ ), in Class II restorations, using stereomicroscope: An in vitro study. Indian J Dent Res. 2019;30(2) 2:77-281. 